\title{
Linguistic Description of Complex Phenomena with the rLDCP R Package
}

\author{
Jose M. Alonso \\ Centro de Investigación en \\ Tecnoloxías da Información (CiTIUS) \\ Patricia Conde-Clemente \\ Universidad de Oviedo, \\ Asturias, Spain \\ University of Santiago de Compostela, \\ Santiago de Compostela, Spain \\ josemaria.alonso.moral@usc.es
}

\author{
Gracian Trivino \\ Phedes Lab, \\ Asturias, Spain
}

\begin{abstract}
Monitoring and analysis of complex phenomena attract the attention of both academy and industry. Dealing with data produced by complex phenomena requires the use of advance computational intelligence techniques. Namely, linguistic description of complex phenomena constitutes a mature research line. It is supported by the Computational Theory of Perceptions grounded on the Fuzzy Sets Theory. Its aim is the development of computational systems with the ability to generate vague descriptions of the world in a similar way how humans do. This is a human-centric and multi-disciplinary research work. Moreover, its success is a matter of careful design; thus, developers play a key role. The rLDCP $\mathrm{R}$ package was designed to facilitate the development of new applications. This demo introduces the use of rLDCP, for both beginners and advance developers, in practical use cases.
\end{abstract}

\section{Introduction}

Trivino and Sugeno (2013) defined a framework for Linguistic Description of Complex Phenomena (LDCP). It is based on the Computational Theory of Perceptions (CTP) introduced by Zadeh (2001) as a new tool for paving the way from computing with numbers to computing with words (Zadeh, 1999). CTP is rooted in the computational intelligence technique best suited to deal with approximate reasoning and vague concepts, i.e., the Fuzzy Sets Theory (Zadeh, 1965; Trillas and Eciolaza, 2015).

LDCP has already been successfully applied in several multi-disciplinary projects. For example:

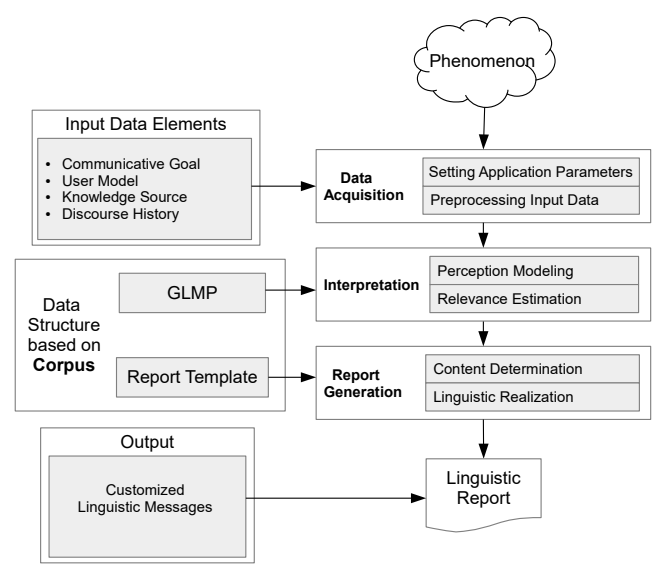

Figure 1: The LDCP Architecture for NLG/D2T.

describing big data (Conde-Clemente et al., 2017b); advising how to save energy at home (CondeClemente et al., 2016); describing physical activity (Sanchez-Valdes et al., 2016); describing drivers' behavior in driving simulations (Eciolaza et al., 2013); or describing double stars in astronomy (Arguelles and Trivino, 2013).

Figure 1 depicts the LDCP architecture for Natural Language Generation in Data-to-text applications (NLG/D2T). It is inspired on the well-known NLG pipeline proposed by Reiter and Dale (2000). The development of new applications with LDCP comprises the following steps:

- Careful analysis of the phenomenon under consideration, regarding: communicative goal, audience background, and the set of natural language expressions (corpus) most commonly used in the context of the application domain. 
- Design of a computational structure (the socalled Granular Linguistic Model of the Phenomenon, GLMP) which organizes all related perceptions in a similar way how humans usually organize their experience by means of natural language.

- Design of a Report Template easy to customize in accordance with the audience requirements.

- Implementation of a computational system able to collect and process raw data, interpret them according to the previously defined GLMP, and producing the Report with the most relevant information to convey to end-users.

Conde-Clemente et al. (2017a) have developed an $\mathrm{R}$ package called $\mathrm{rLDCP}^{1}$ which constitutes a first implementation in $\mathrm{R}$ of the steps enumerated above. Thus, it facilitates the use of the LDCP architecture in new applications.

\section{Structure of the Demo}

This demo describes how to use rLDCP from scratch. Firstly, we explain how to download and install rLDCP. Secondly, we detail how to run step by step the toy example ComfortableRoom from the point of view of beginners and advance developers. The goal is describing the comfort in a room with respect to temperature and light intensity data values previously stored in a ".csv" file.

Then, we show how to use rLDCP for building a real application: The inProfilePhoto mobile app. We implement with rLDCP the application described in (Conde-Clemente et al., 2013) where an NLG system guided a person with visual disabilities to take his/her own profile photos.

\section{Acknowledgments}

This work is supported by the Spanish Ministry of Economy and Competitiveness [grant numbers TIN2014-56633-C3-3-R, TIN2014-56633-C3-1-R]; the "Consellería de Cultura, Educación e Ordenación Universitaria" (accreditation 2016-2019, ED431G/08) and the European Regional Development Fund (ERDF); and the Spanish Ministry of

\footnotetext{
${ }^{1} \mathrm{rLDCP}$ is an R package for text generation from data. It is freely available at [http://www.phedes.com/rLDCP]
}

Science and Innovation [grant number FPI-MICINN BES-2012-057427].

\section{References}

Luis Arguelles and Gracian Trivino. 2013. I-struve: Automatic linguistic descriptions of visual double stars. Engineering Applications of Artificial Intelligence, 26(9):2083-2092.

Patricia Conde-Clemente, Jose M. Alonso, and Gracian Trivino. 2013. Interpretable fuzzy system allowing to be framed in a profile photo through linguistic expressions. In Proceedings of 8th Conference of the European Society for Fuzzy Logic and Technology (EUSFLAT), pages 463-468, Milano, Italy.

Patricia Conde-Clemente, Jose M. Alonso, and Gracian Trivino. 2016. Towards automatic generation of linguistic advice for saving energy at home. Soft Computing, pages 1-15.

Patricia Conde-Clemente, Jose M. Alonso, and Gracian Trivino. 2017a. rldcp: R package for text generation from data. In IEEE International Conference on Fuzzy Systems (FUZZ-IEEE), pages 1-6, Naples, Italy.

Patricia Conde-Clemente, Gracian Trivino, and Jose M. Alonso. 2017b. Generating automatic linguistic descriptions with big data. Information Sciences, 380:12-30.

Luka Eciolaza, Martin Pereira-Fariña, and Gracian Trivino. 2013. Automatic linguistic reporting in driving simulation environments. Applied Soft Computing, 13(9):3956-3967.

Ehud Reiter and Robert Dale. 2000. Building natural language generation systems, volume 33. MIT Press.

Daniel Sanchez-Valdes, Alberto Alvarez-Alvarez, and Gracian Trivino. 2016. Dynamic linguistic descriptions of time series applied to self-track the physical activity. Fuzzy Sets and Systems, 285:162-181.

Enric Trillas and Luka Eciolaza. 2015. Fuzzy Logic: An Introductory Course for Engineering Students. Springer.

Gracian Trivino and Michio Sugeno. 2013. Towards linguistic descriptions of phenomena. International Journal of Approximate Reasoning, 54(1):22-34.

Lotfi A. Zadeh. 1965. Fuzzy sets. Information and Control, 8(3):338-353.

Lotfi A. Zadeh. 1999. From computing with numbers to computing with words. from manipulation of measurements to manipulation of perceptions. IEEE Transactions on Circuits and Systems, 46(1):105-119.

Lotfi A. Zadeh. 2001. A new direction in AI: Toward a computational theory of perceptions. Artificial Intelligent Magazine, 22(1):73-84. 